\title{
Insulin minimises cell injury in an ex vivo perfused rat liver model
}

\author{
Cuttaia Héléna, Nuyens Vincent, Boogaerts Jean, Papegay Bérengère Laboratory of Experimental \\ Medicine, University Hospital Centre of Charleroi, Belgium
}

Introduction: In the field of organ preservation, various solutions exist according to the composition of electrolytes, buffers, antioxydants and energy precursors. Insulin is added to University of Wisconsin preservation solution with the aim of stimulating glycolysis (1). Nevertheless, previous studies have shown that insulin exacerbates the ischemic injury in rat liver transplantation $(2,3)$. The value of insulin in preservation solution therefore remains unclear. The aim of this study was to determine the effect of insulin on preservation of an ex vivo glucose-free perfused rat liver.

Materials and Methods: After Animal Care Committee approval, Wistar rats were fasted for 16 hrs. After they were anaesthetised,

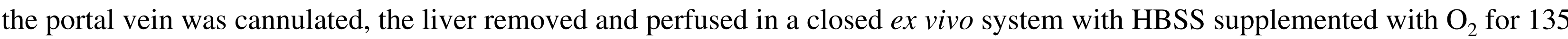
min. The animals were divided into 3 groups $(n=5)$ : control and insulin groups in which medium contains respectively 35 and 70 IU/L insulin. Glucose, lactate, potassium, and enzymes were analysed in perfusate samples at different time-points. The proportion of glycogen in hepatocytes and energy charge $(\mathrm{EC}=[\mathrm{ATP}]+1: 2[\mathrm{ADP}] /[\mathrm{ATP}]+[\mathrm{ADP}]+[\mathrm{AMP}])$ were determined in tissue biopsies. Mean $\pm \mathrm{SD}$. ANOVA and $\mathrm{Chi}^{2}$ tests.

\section{Results}

Results are displayed in the figures below.
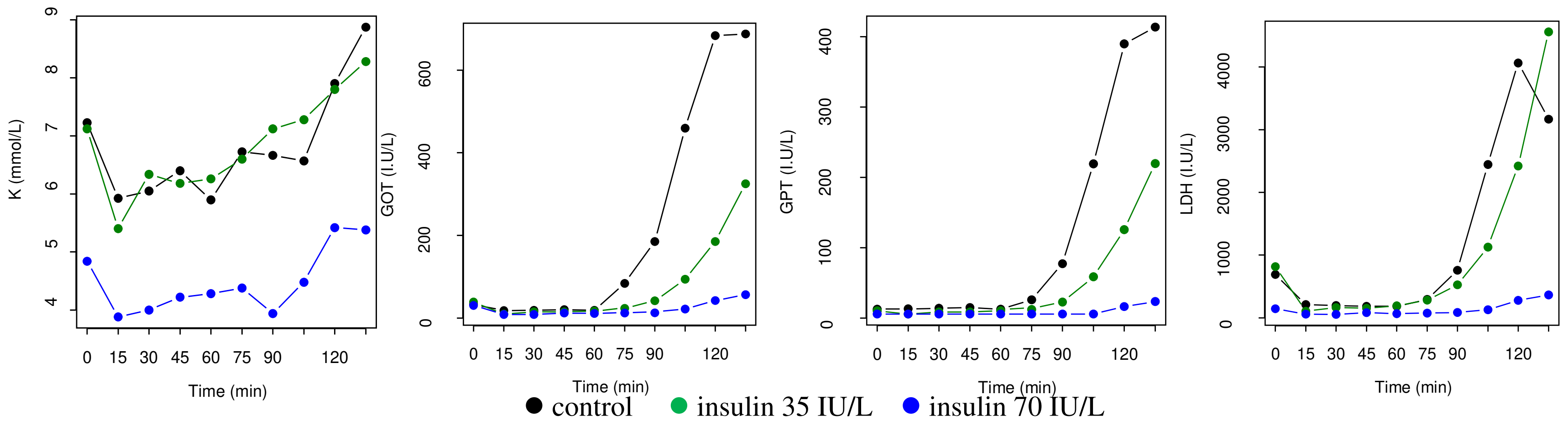

Variables at 135 min are displayed in the table, The concentrations of potassium and enzymes were lower in both insulin groups (P $<0.01)$.

\begin{tabular}{lcccc}
\hline Variable & Control & Insulin 35 IU/L & Insulin 70 IU/L & $P$-value \\
\hline Glucose (mg/dl) & $7.50 \pm 1.91$ & $11.20 \pm 7.33$ & $25.40 \pm 18.09$ & 0.092 \\
Lactate (mmol/L) & $0.38 \pm 0.17$ & $0.46 \pm 0.19$ & $1.06 \pm 0.96$ & 0.14 \\
Potassium (mEq/L) & $8.88 \pm 1.34$ & $8.28 \pm 0.57$ & $5.38 \pm 1.31$ & $<0.001$ \\
GOT (IU/L) & $687.75 \pm 787.02$ & $324.80 \pm 234.69$ & $57.00 \pm 51.49$ & 0.007 \\
GPT (UI/L) & $413.75 \pm 354.37$ & $219.60 \pm 213.81$ & $23.60 \pm 39.35$ & 0.002 \\
LDH (UI/L) & $4,065.3 \pm 4533.1$ & $2,421.8 \pm 1839.6$ & $277.80 \pm 290.87$ & 0.004 \\
Glycogène (\%) & $7.58 \pm 1.43$ & $10.47 \pm 4.77$ & $21.22 \pm 27.80$ & 0.19 \\
Energetic charge & $0.10 \pm 0.04$ & $0.15 \pm 0.04$ & $0.17 \pm 0.08$ & 0.69 \\
\hline
\end{tabular}

Discussion: Results of the present experiment show that insulin minimises rat liver injury during ex vivo perfusion. In essence, insulin accelerates glycolysis, a source of ATP, and simulates glycogen synthesis (1). We observed a slight but not significant increase in EC and glycogen concentration in hepatocytes. Such findings seem to indicate that livers perfused with insulin in a glucose-free buffer solution were metabolically more active at the end of the preservation period.

References: 1. Biguzas et al. Transplantation 1990;49:1051-5; 2. Yu et al Transplantation 1990;49:1060-6; 